Supplement of Atmos. Chem. Phys., 18, 185-202, 2018

https://doi.org/10.5194/acp-18-185-2018-supplement

(C) Author(s) 2018. This work is distributed under

the Creative Commons Attribution 3.0 License.

(c) (1)

Supplement of

\title{
Estimating regional-scale methane flux and budgets using CARVE aircraft measurements over Alaska
}

\section{Sean Hartery et al.}

Correspondence to: Rachel Y.-W. Chang (rachel.chang@dal.ca)

The copyright of individual parts of the supplement might differ from the CC BY 3.0 License. 


\section{S1 STILT footprint sensitivities}

The STILT simulations assume that particles in the lower $50 \%$ of the boundary layer are directly influenced by the surface. A previous sensitivity study found that varying this percentage from $10-100 \%$ did not change the results significantly, although a lower value resulted in fewer particles actually influenced by the surface and an increase in noise (Gerbig et al., 2003). It should be noted that this sensitivity study was conducted over continental North America using different meteorological drivers and that these results may not be valid over our study domain, especially in the spring and fall. Nevertheless, it suggests that this assumption is not the major contributor to uncertainties in our results.

\section{S2 Individual $\mathrm{CH}_{4}$ profiles}

The following Tables include the regional $\mathrm{CH}_{4}$ flux estimations of Hartery et al. (2017) in tabulated form. Please refer to the main text for specific details such as the domain over which these estimates are valid and the measurements and particle transport modelling from which they were derived. Observer information is listed for all those profiles for which the estimate was determined to be unsuitable for analysis in three different messages. The first, "Large variability in $\mathrm{FT} \mathrm{CH}_{4}$," refers to an observed problem in constraining the background value of $\mathrm{CH}_{4}$ in the free troposphere which is used to calculate the mixed layer enhancement of $\mathrm{CH}_{4}$ (and subsequently the regional $\mathrm{CH}_{4}$ flux). Since our method involves integrating observed profiles of mole fractions of $\mathrm{CH}_{4}$ over the entire mixed layer, a large uncertainty in the background will result in a large uncertainty in $\mathrm{CH}_{4}$ flux and therefore these profiles were withheld from further analysis.

The second observer message is, "Difficulty estimating BLH." This message is reported when the refractivity method for determining the mixed layer height significantly differed from observations of the profiles of water vapour, potential temperature and other trace gasses. It also appears when the boundary layer height predicted from modelling simulations differed from observations.

The third message which appears throughout these tables reports that "BL influence anomalously low." This message was used to indicate when the average integrated footprint influence throughout the mixed layer was less than $5 \%$ of the campaign average $\left(\bar{I} \sim 1 \mathrm{ppm} \mathrm{nmol}^{-1} \mathrm{~m}^{-2} \mathrm{~s}^{-1}\right)$. When footprint influence is so low it is indicative that the air mass we measured was not predominantly of local origin and therefore not useful in deriving regional $\mathrm{CH}_{4}$ estimates. This also results in overestimates of $\mathrm{CH}_{4}$ flux as a consequence of our estimation method (see main text). The resulting profiles kept for analysis have been marked in the final column of each table by "incl."
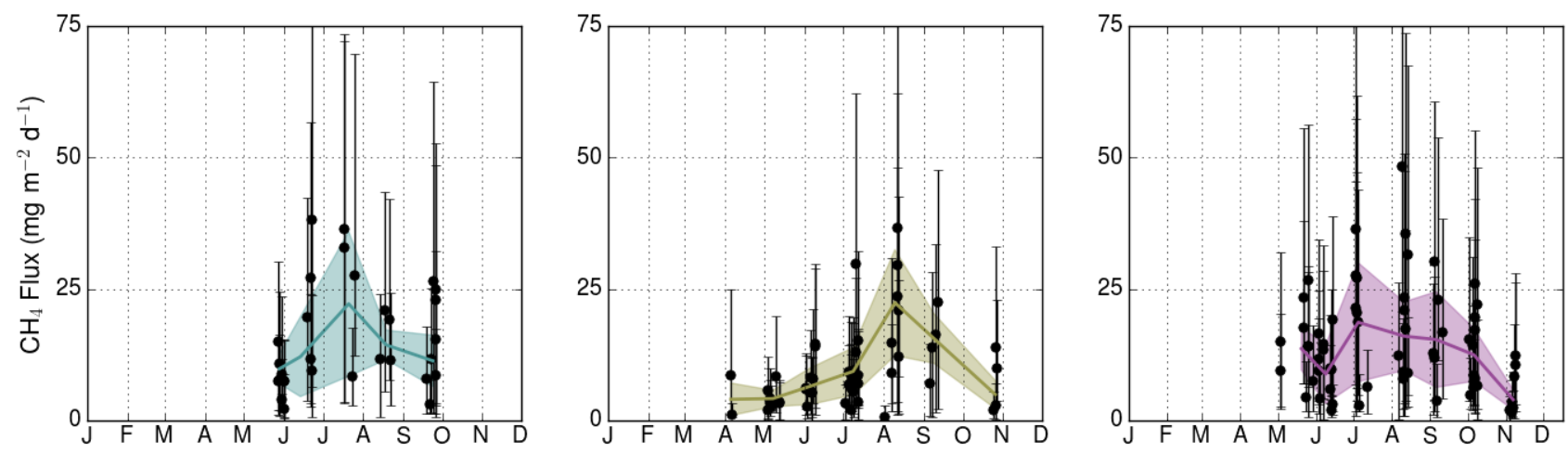

Figure S1: Individual estimates of $\mathrm{CH}_{4}$ flux that were used in the analysis of the main text are shown overlaying the monthly average $\mathrm{CH}_{4}$ fluxes. Error bars reflect uncertainties in individual flux estimates derived from Monte Carlo/bootstrap estimation.

As there can be considerable monthly variability among $\mathrm{CH}_{4}$ flux estimates within a given month, we plot individual net flux estimates over the monthly averages. As such, Fig. S1 is a useful summary of Tables S1-S3; n.b., only profiles included in the analysis are plotted. To highlight the fact that overall, the individual $\mathrm{CH}_{4}$ flux estimates were normally distributed about the monthly mean $\mathrm{CH}_{4}$ flux estimates, the residuals of 
each individual estimate were plotted in a histogram in Fig. S2. The shape of this distribution gives us confidence that our monthly averaging is representative of the underlying seasonal trend.

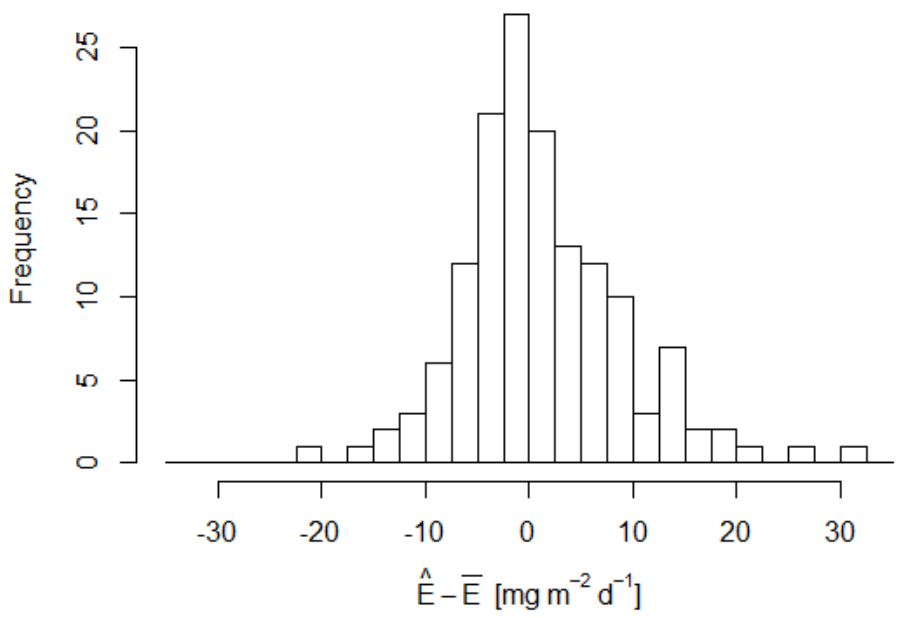

Figure S2: Residuals of individual $\mathrm{CH}_{4}$ flux estimates with respect to monthly averages are shown in the histogram. 
Table S1: CARVE 2012 regionally averaged $\mathrm{CH}_{4}$ Fluxes are presented.

\begin{tabular}{|c|c|c|c|c|}
\hline YYYYMMDD & Profile \# & Flux $\left(m g m^{-2} d^{-1}\right)$ & Observer Information & Final Analysis \\
\hline 20120523 & 1 & 0.8 & Large variability in $\mathrm{FT} \mathrm{CH}_{4}$ & - \\
\hline 20120527 & 1 & 5.0 & Difficulty estimating BLH & - \\
\hline 20120527 & 2 & 7.4 & & incl. \\
\hline 20120527 & 3 & 15.0 & & incl. \\
\hline 20120528 & 1 & 10.9 & & incl. \\
\hline 20120528 & 2 & 7.8 & & incl. \\
\hline 20120530 & 1 & 8.9 & & incl. \\
\hline 20120530 & 2 & 4.1 & & incl. \\
\hline 20120530 & 3 & 4.6 & Large variability in $\mathrm{FT} \mathrm{CH}_{4}$ & - \\
\hline 20120601 & 1 & 7.6 & & incl. \\
\hline 20120601 & 2 & 7.5 & & incl. \\
\hline 20120601 & 3 & 2.2 & & incl. \\
\hline 20120618 & 1 & 1.8 & Large variability in $\mathrm{FT} \mathrm{CH}_{4}$ & - \\
\hline 20120618 & 2 & 49.0 & Large variability in $\mathrm{FT} \mathrm{CH}_{4}$ & - \\
\hline 20120618 & 3 & 8.3 & Large variability in $\mathrm{FT} \mathrm{CH}_{4}$ & - \\
\hline 20120619 & 1 & 2.7 & Large variability in $\mathrm{FT} \mathrm{CH}_{4}$ & - \\
\hline 20120619 & 2 & 19.6 & & incl. \\
\hline$\cdots$ & .. & $\ldots$ & $\cdots$ & $\cdots$ \\
\hline
\end{tabular}


Table S1: (cont.)

\begin{tabular}{|c|c|c|c|c|}
\hline YYYYMMDD & Profile \# & Flux $\left(\mathrm{mg} \mathrm{m}^{-2} \mathrm{~d}^{-1}\right)$ & Observer Information & Final Analysis \\
\hline$\ldots$ & $\ldots$ & $\ldots$ & $x^{2}+\ldots$ & $\ldots$ \\
\hline 20120619 & 3 & 9.1 & BL influence anomalously low, Large variability in $\mathrm{FT} \mathrm{CH}_{4}$ & - \\
\hline 20120621 & 1 & 27.1 & & incl. \\
\hline 20120621 & 2 & 11.7 & & incl. \\
\hline 20120621 & 3 & 3.9 & Difficulty estimating BLH & - \\
\hline 20120622 & 1 & -6.2 & Large variability in $\mathrm{FT}^{\mathrm{CH}_{4}}$ & - \\
\hline 20120622 & 2 & 43.1 & Difficulty estimating BLH, BL influence anomalously low & - \\
\hline 20120622 & 3 & 38.1 & & incl. \\
\hline 20120622 & 4 & 9.5 & & incl. \\
\hline 20120624 & 1 & -3.4 & Large variability in $\mathrm{FT}_{\mathrm{CH}_{4}}$ & - \\
\hline 20120624 & 2 & 25.7 & Difficulty estimating BLH, BL influence anomalously low & - \\
\hline 20120717 & 1 & 32.8 & & incl. \\
\hline 20120717 & 2 & 31.0 & Difficulty estimating BLH & - \\
\hline 20120717 & 3 & 36.3 & & incl. \\
\hline 20120722 & 1 & -7.9 & Large variability in $\mathrm{FT}_{\mathrm{CH}_{4}}$ & - \\
\hline 20120722 & 2 & 7.3 & Large variability in $\mathrm{FT} \mathrm{CH}_{4}$ & - \\
\hline 20120722 & 3 & -1.4 & Large variability in $\mathrm{FT} \mathrm{CH}_{4}$ & - \\
\hline$\ldots$ & $\ldots$ & $\ldots$ & $\ldots$ & $\cdots$ \\
\hline
\end{tabular}


Table S1: (cont.)

\begin{tabular}{|c|c|c|c|c|}
\hline YYYYMMDD & Profile \# & Flux $\left(\mathrm{mg} \mathrm{m}^{-2} \mathrm{~d}^{-1}\right)$ & Observer Information & Final Analysis \\
\hline$\ldots$ & $\ldots$ & $\ldots$ & $\ldots$ & $\ldots$ \\
\hline 20120724 & 1 & 8.3 & & incl. \\
\hline 20120724 & 2 & 3.6 & Large variability in $\mathrm{FT} \mathrm{CH}_{4}$ & - \\
\hline 20120725 & 1 & 3.7 & Large variability in $\mathrm{FT} \mathrm{CH}_{4}$ & - \\
\hline 20120725 & 2 & 11.8 & Large variability in $\mathrm{FT} \mathrm{CH}_{4}$ & - \\
\hline 20120725 & 3 & 27.5 & & incl. \\
\hline 20120814 & 1 & 15.5 & Large variability in $\mathrm{FT} \mathrm{CH}_{4}$ & - \\
\hline 20120814 & 2 & 11.7 & & incl. \\
\hline 20120818 & 1 & 20.9 & & incl. \\
\hline 20120819 & 1 & -19.0 & BL influence anomalously low, Large variability in $\mathrm{FT} \mathrm{CH}_{4}$ & - \\
\hline 20120819 & 2 & 8.2 & Large variability in $\mathrm{FT} \mathrm{CH}_{4}$ & - \\
\hline 20120819 & 3 & -4.0 & Difficulty estimating BLH & - \\
\hline 20120820 & 1 & 3.5 & Large variability in $\mathrm{FT} \mathrm{CH}_{4}$ & - \\
\hline 20120821 & 1 & 19.1 & & incl. \\
\hline 20120821 & 2 & 19.2 & Difficulty estimating BLH & - \\
\hline 20120822 & 1 & 11.4 & & incl. \\
\hline 20120823 & 1 & 18.5 & Large variability in $\mathrm{FT} \mathrm{CH}_{4}$ & - \\
\hline$\ldots$ & $\ldots$ & $\ldots$ & $\cdots$ & $\ldots$ \\
\hline
\end{tabular}


Table S1: (cont.)

\begin{tabular}{|c|c|c|c|c|}
\hline YYYYMMDD & Profile \# & Flux $\left(\mathrm{mg} \mathrm{m}^{-2} \mathrm{~d}^{-1}\right)$ & Observer Information & Final Analysis \\
\hline$\ldots$ & $\ldots$ & $\ldots$ & $\ldots$ & $\ldots$ \\
\hline 20120919 & 1 & 7.9 & & incl. \\
\hline 20120921 & 1 & 2.6 & Difficulty estimating BLH & - \\
\hline 20120921 & 2 & 3.2 & & incl. \\
\hline 20120922 & 1 & 11.7 & & incl. \\
\hline 20120923 & 1 & -77.2 & Difficulty estimating BLH, BL influence anomalously low & - \\
\hline 20120924 & 1 & 26.4 & & incl. \\
\hline 20120924 & 2 & 53.3 & Large variability in $\mathrm{FT} \mathrm{CH}_{4}$ & - \\
\hline 20120926 & 1 & 8.6 & & incl. \\
\hline 20120926 & 2 & 24.8 & & incl. \\
\hline 20120926 & 3 & 15.4 & & incl. \\
\hline 20120926 & 4 & 22.9 & & incl. \\
\hline
\end{tabular}

Table S2: CARVE 2013 regionally averaged $\mathrm{CH}_{4}$ Fluxes are presented.

\begin{tabular}{|c|c|c|c|c|}
\hline YYYYMMDD & Profile \# & Flux $\left(\mathrm{mg} \mathrm{m}^{-2} \mathrm{~d}^{-1}\right)$ & Observer Information & Final Analysis \\
\hline 20130402 & 1 & 2.0 & Large variability in $\mathrm{FT} \mathrm{CH}_{4}$ & - \\
\hline 20130403 & 1 & 0.6 & Large variability in $\mathrm{FT} \mathrm{CH}_{4}$ & - \\
\hline 20130404 & 1 & 18.4 & Large variability in $\mathrm{FT} \mathrm{CH}_{4}$ & - \\
\hline 20130404 & 2 & 83.8 & BL influence anomalously low, Large variability in $\mathrm{FT}^{\mathrm{CH}_{4}}$ & - \\
\hline 20130404 & 3 & 28.4 & Large variability in $\mathrm{FT} \mathrm{CH}_{4}$ & - \\
\hline 20130405 & 1 & 8.7 & & incl. \\
\hline 20130406 & 1 & 4.2 & Difficulty estimating BLH & - \\
\hline 20130406 & 2 & 2.9 & Large variability in $\mathrm{FT} \mathrm{CH}_{4}$ & - \\
\hline 20130406 & 3 & 1.0 & & incl. \\
\hline 20130502 & 1 & 10.9 & Difficulty estimating BLH & - \\
\hline 20130502 & 2 & 4.2 & Difficulty estimating BLH & - \\
\hline 20130504 & 1 & 2.1 & & incl. \\
\hline 20130504 & 2 & 5.7 & & incl. \\
\hline 20130506 & 1 & 3.4 & & incl. \\
\hline 20130506 & 2 & 4.2 & & incl. \\
\hline 20130507 & 1 & 2.6 & & incl. \\
\hline 20130507 & 2 & 1.0 & Large variability in $\mathrm{FT} \mathrm{CH}_{4}$ & - \\
\hline$\cdots$ & $\cdots$ & $\cdots$ & $\ldots$ & $\cdots$ \\
\hline
\end{tabular}


Table S2: (cont.)

\begin{tabular}{|c|c|c|c|c|}
\hline YYYYMMDD & Profile \# & Flux $\left(\mathrm{mg} \mathrm{m}^{-2} \mathrm{~d}^{-1}\right)$ & Observer Information & Final Analysis \\
\hline$\ldots$ & $\ldots$ & $\ldots$ & $\ldots$ & $\ldots$ \\
\hline 20130507 & 3 & -4.5 & Large variability in $\mathrm{FT} \mathrm{CH}_{4}$ & - \\
\hline 20130508 & 1 & 2.2 & Difficulty estimating BLH & - \\
\hline 20130509 & 1 & -9.6 & Large variability in $\mathrm{FT} \mathrm{CH}_{4}$ & - \\
\hline 20130509 & 2 & -0.4 & Large variability in $\mathrm{FT} \mathrm{CH}_{4}$ & - \\
\hline 20130509 & 3 & 16.0 & Large variability in $\mathrm{FT} \mathrm{CH}_{4}$ & - \\
\hline 20130510 & 1 & 8.4 & & incl. \\
\hline 20130510 & 2 & 8.1 & Large variability in $\mathrm{FT} \mathrm{CH}_{4}$ & - \\
\hline 20130513 & 1 & 3.5 & & incl. \\
\hline 20130513 & 2 & 3.4 & & incl. \\
\hline 20130602 & 1 & 6.1 & & incl. \\
\hline 20130602 & 2 & 6.0 & & incl. \\
\hline 20130603 & 1 & 2.8 & & incl. \\
\hline 20130603 & 2 & 2.1 & Large variability in $\mathrm{FT} \mathrm{CH}_{4}$ & - \\
\hline 20130606 & 1 & 8.1 & & incl. \\
\hline 20130606 & 2 & 0.2 & Difficulty estimating BLH & - \\
\hline 20130607 & 1 & 5.2 & & incl. \\
\hline$\cdots$ & $\cdots$ & $\cdots$ & $\cdots$ & $\cdots$ \\
\hline
\end{tabular}


Table S2: (cont.)

\begin{tabular}{|c|c|c|c|c|}
\hline YYYYMMDD & Profile \# & Flux $\left(\mathrm{mg} \mathrm{m}^{-2} \mathrm{~d}^{-1}\right)$ & Observer Information & Final Analysis \\
\hline$\ldots$ & $\ldots$ & $\ldots$ & $\ldots$ & $\ldots$ \\
\hline 20130607 & 2 & 5.3 & & incl. \\
\hline 20130607 & 3 & 0.3 & Difficulty estimating BLH & - \\
\hline 20130608 & 1 & 8.0 & & incl. \\
\hline 20130609 & 1 & 14.1 & & incl. \\
\hline 20130609 & 2 & 14.5 & & incl. \\
\hline 20130611 & 1 & 3.4 & Difficulty estimating BLH & - \\
\hline 20130611 & 2 & 8.8 & BL influence anomalously low, Large variability in $\mathrm{FT} \mathrm{CH}_{4}$ & - \\
\hline 20130611 & 3 & -86.6 & Difficulty estimating BLH, BL influence anomalously low & - \\
\hline 20130703 & 1 & 3.2 & & incl. \\
\hline 20130703 & 2 & -3.1 & Difficulty estimating BLH & - \\
\hline 20130704 & 1 & 3.0 & Difficulty estimating BLH & - \\
\hline 20130705 & 1 & 9.3 & & incl. \\
\hline 20130705 & 2 & 6.8 & & incl. \\
\hline 20130705 & 3 & 0.6 & Large variability in $\mathrm{FT} \mathrm{CH}_{4}$ & - \\
\hline 20130707 & 1 & 8.5 & & incl. \\
\hline 20130707 & 2 & -3.2 & Large variability in $\mathrm{FT} \mathrm{CH}_{4}$ & - \\
\hline$\ldots$ & $\ldots$ & $\ldots$ & $\cdots$ & $\ldots$ \\
\hline
\end{tabular}


Table S2: (cont.)

\begin{tabular}{|c|c|c|c|c|}
\hline YYYYMMDD & Profile \# & Flux $\left(m g m^{-2} d^{-1}\right)$ & Observer Information & Final Analysis \\
\hline$\ldots$ & $\ldots$ & $\ldots$ & $\ldots$ & $\ldots$ \\
\hline 20130707 & 3 & 2.0 & & incl. \\
\hline 20130707 & 4 & 7.2 & & incl. \\
\hline 20130707 & 5 & 6.2 & & incl. \\
\hline 20130709 & 1 & 9.4 & & incl. \\
\hline 20130709 & 2 & 5.5 & & incl. \\
\hline 20130709 & 3 & 8.7 & & incl. \\
\hline 20130711 & 1 & 15.8 & Difficulty estimating BLH & - \\
\hline 20130711 & 2 & 29.9 & & incl. \\
\hline 20130711 & 3 & 13.0 & & incl. \\
\hline 20130712 & 1 & 3.6 & & incl. \\
\hline 20130712 & 2 & 8.7 & & incl. \\
\hline 20130712 & 3 & 7.2 & & incl. \\
\hline 20130712 & 4 & 15.1 & & incl. \\
\hline 20130802 & 1 & 0.7 & & incl. \\
\hline 20130802 & 2 & 6.0 & Difficulty estimating BLH & - \\
\hline 20130803 & 1 & 6.1 & Difficulty estimating BLH, BL influence anomalously low & - \\
\hline$\cdots$ & $\cdots$ & $\ldots$ & $\cdots$ & $\cdots$ \\
\hline
\end{tabular}


Table S2: (cont.)

\begin{tabular}{|c|c|c|c|c|}
\hline YYYYMMDD & Profile \# & Flux $\left(\mathrm{mg} \mathrm{m}^{-2} \mathrm{~d}^{-1}\right)$ & Observer Information & Final Analysis \\
\hline$\ldots$ & $\ldots$ & $\ldots$ & $\ldots$ & $\ldots$ \\
\hline 20130803 & 2 & 13.3 & Large variability in $\mathrm{FT} \mathrm{CH}_{4}$ & - \\
\hline 20130803 & 3 & -0.5 & Large variability in $\mathrm{FT} \mathrm{CH}_{4}$ & - \\
\hline 20130804 & 1 & 5.3 & Large variability in $\mathrm{FT} \mathrm{CH}_{4}$ & - \\
\hline 20130807 & 1 & -14.7 & BL influence anomalously low, Large variability in $\mathrm{FT} \mathrm{CH}_{4}$ & - \\
\hline 20130807 & 2 & 9.0 & & incl. \\
\hline 20130807 & 3 & 14.9 & & incl. \\
\hline 20130811 & 1 & 9.8 & BL influence anomalously low, Large variability in $\mathrm{FT} \mathrm{CH}_{4}$ & - \\
\hline 20130811 & 2 & 25.2 & Large variability in $\mathrm{FT} \mathrm{CH}_{4}$ & - \\
\hline 20130811 & 3 & 16.8 & Large variability in $\mathrm{FT}_{\mathrm{CH}_{4}}$ & - \\
\hline 20130812 & 1 & 23.7 & & incl. \\
\hline 20130812 & 2 & 36.7 & & incl. \\
\hline 20130812 & 3 & 29.6 & & incl. \\
\hline 20130813 & 1 & 41.9 & Large variability in $\mathrm{FT} \mathrm{CH}_{4}$ & - \\
\hline 20130813 & 2 & 20.8 & & incl. \\
\hline 20130813 & 3 & 12.2 & & incl. \\
\hline 20130905 & 1 & -0.4 & Large variability in $\mathrm{FT} \mathrm{CH}_{4}$ & - \\
\hline$\ldots$ & $\ldots$ & $\ldots$ & $\cdots$ & $\cdots$ \\
\hline
\end{tabular}


Table S2: (cont.)

\begin{tabular}{|c|c|c|c|c|}
\hline YYYYMMDD & Profile \# & Flux $\left(\mathrm{mg} \mathrm{m}^{-2} \mathrm{~d}^{-1}\right)$ & Observer Information & Final Analysis \\
\hline$\ldots$ & $\ldots$ & $\ldots$ & $\ldots$ & $\ldots$ \\
\hline 20130906 & 1 & 3.3 & Large variability in $\mathrm{FT} \mathrm{CH}_{4}$ & - \\
\hline 20130906 & 2 & 6.9 & & incl. \\
\hline 20130906 & 3 & 11.7 & Difficulty estimating BLH & - \\
\hline 20130907 & 1 & 13.9 & & incl. \\
\hline 20130910 & 1 & 11.4 & Difficulty estimating BLH & - \\
\hline 20130910 & 2 & 16.4 & & incl. \\
\hline 20130912 & 1 & 22.5 & & incl. \\
\hline 20131024 & 1 & 47.0 & Large variability in $\mathrm{FT} \mathrm{CH}_{4}$ & - \\
\hline 20131024 & 2 & 14.3 & Large variability in $\mathrm{FT} \mathrm{CH}_{4}$ & - \\
\hline 20131025 & 1 & 2.0 & & incl. \\
\hline 20131026 & 1 & 13.8 & & incl. \\
\hline 20131026 & 2 & 2.8 & & incl. \\
\hline 20131027 & 1 & 10.0 & & incl. \\
\hline 20131027 & 2 & 7.2 & Difficulty estimating BLH, BL influence anomalously low & - \\
\hline
\end{tabular}


Table S3: CARVE 2014 regionally averaged $\mathrm{CH}_{4}$ Fluxes are presented.

\begin{tabular}{|c|c|c|c|c|}
\hline YYYYMMDD & Profile \# & Flux $\left(\mathrm{mg} \mathrm{m}^{-2} \mathrm{~d}^{-1}\right)$ & Observer Information & Final Analysis \\
\hline 20140503 & 1 & 15.1 & & incl. \\
\hline 20140503 & 2 & -7.8 & Large variability in $\mathrm{FT} \mathrm{CH}_{4}$ & - \\
\hline 20140503 & 3 & 9.4 & & incl. \\
\hline 20140522 & 1 & 23.3 & & incl. \\
\hline 20140522 & 2 & 17.7 & & incl. \\
\hline 20140523 & 1 & 35.8 & Large variability in $\mathrm{FT} \mathrm{CH}_{4}$ & - \\
\hline 20140524 & 1 & 12.0 & Difficulty estimating BLH & - \\
\hline 20140524 & 2 & 4.4 & & incl. \\
\hline 20140526 & 1 & -0.7 & Difficulty estimating BLH & - \\
\hline 20140526 & 2 & 14.0 & & incl. \\
\hline 20140526 & 3 & 14.2 & & incl. \\
\hline 20140526 & 4 & 26.7 & & incl. \\
\hline 20140529 & 1 & 7.5 & & incl. \\
\hline 20140603 & 1 & 11.7 & & incl. \\
\hline 20140603 & 2 & 9.5 & & incl. \\
\hline 20140603 & 3 & 16.5 & & incl. \\
\hline 20140604 & 1 & 4.3 & & incl. \\
\hline$\cdots$ & $\cdots$ & $\ldots$ & $\cdots$ & $\cdots$ \\
\hline
\end{tabular}


Table S3: (cont.)

\begin{tabular}{|c|c|c|c|c|}
\hline YYYYMMDD & Profile \# & Flux $\left(m g m^{-2} d^{-1}\right)$ & Observer Information & Final Analysis \\
\hline$\ldots$ & $\ldots$ & $\ldots$ & $\ldots$ & $\ldots$ \\
\hline 20140607 & 1 & 13.4 & & incl. \\
\hline 20140607 & 2 & 14.5 & & incl. \\
\hline 20140607 & 3 & 0.2 & Large variability in $\mathrm{FT} \mathrm{CH}_{4}$ & - \\
\hline 20140611 & 1 & 2.0 & Difficulty estimating BLH & - \\
\hline 20140611 & 2 & 2.6 & Difficulty estimating BLH & - \\
\hline 20140611 & 3 & 1.8 & Large variability in $\mathrm{FT} \mathrm{CH}_{4}$ & - \\
\hline 20140612 & 1 & 5.9 & & incl. \\
\hline 20140612 & 2 & 9.8 & & incl. \\
\hline 20140613 & 1 & 3.4 & Difficulty estimating BLH & - \\
\hline 20140613 & 2 & 1.9 & & incl. \\
\hline 20140613 & 3 & 9.6 & & incl. \\
\hline 20140614 & 1 & -1.2 & Large variability in $\mathrm{FT} \mathrm{CH}_{4}$ & - \\
\hline 20140614 & 2 & 19.2 & & incl. \\
\hline 20140614 & 3 & 3.1 & & incl. \\
\hline 20140703 & 1 & 3.5 & Difficulty estimating BLH & - \\
\hline 20140703 & 2 & 36.3 & & incl. \\
\hline$\ldots$ & $\cdots$ & $\ldots$ & $\cdots$ & $\ldots$ \\
\hline
\end{tabular}


Table S3: (cont.)

\begin{tabular}{|c|c|c|c|c|}
\hline YYYYMMDD & Profile \# & Flux $\left(\mathrm{mg} \mathrm{m}^{-2} \mathrm{~d}^{-1}\right)$ & Observer Information & Final Analysis \\
\hline$\ldots$ & $\ldots$ & $\ldots$ & $\ldots$ & $\ldots$ \\
\hline 20140703 & 3 & 27.6 & & incl. \\
\hline 20140703 & 4 & 21.3 & & incl. \\
\hline 20140704 & 1 & 27.0 & & incl. \\
\hline 20140704 & 2 & 20.5 & & incl. \\
\hline 20140705 & 1 & 5.0 & Large variability in $\mathrm{FT} \mathrm{CH}_{4}$ & - \\
\hline 20140705 & 2 & 18.8 & & incl. \\
\hline 20140705 & 3 & -1.2 & Difficulty estimating BLH & - \\
\hline 20140706 & 1 & 2.9 & & incl. \\
\hline 20140706 & 2 & 3.1 & Difficulty estimating BLH & - \\
\hline 20140706 & 3 & 12.9 & Difficulty estimating BLH & - \\
\hline 20140710 & 1 & 0.3 & Large variability in $\mathrm{FT} \mathrm{CH}_{4}$ & - \\
\hline 20140711 & 1 & 1.1 & Large variability in $\mathrm{FT} \mathrm{CH}_{4}$ & - \\
\hline 20140712 & 1 & 6.4 & & incl. \\
\hline 20140712 & 2 & 6.5 & Difficulty estimating BLH & - \\
\hline 20140806 & 1 & 14.6 & Difficulty estimating BLH & - \\
\hline 20140806 & 2 & 12.4 & & incl. \\
\hline$\ldots$ & $\ldots$ & $\cdots$ & $\cdots$ & $\ldots$ \\
\hline
\end{tabular}


Table S3: (cont.)

\begin{tabular}{|c|c|c|c|c|}
\hline YYYYMMDD & Profile \# & Flux $\left(\mathrm{mg} \mathrm{m}^{-2} \mathrm{~d}^{-1}\right)$ & Observer Information & Final Analysis \\
\hline$\ldots$ & $\ldots$ & $\ldots$ & $\ldots$ & $\ldots$ \\
\hline 20140809 & 1 & 48.3 & & incl. \\
\hline 20140809 & 2 & 24.6 & Difficulty estimating BLH & - \\
\hline 20140810 & 1 & 1.0 & Difficulty estimating BLH & - \\
\hline 20140810 & 2 & 9.3 & & incl. \\
\hline 20140810 & 3 & 8.0 & & incl. \\
\hline 20140811 & 1 & 20.9 & & incl. \\
\hline 20140811 & 2 & 23.5 & & incl. \\
\hline 20140812 & 1 & 47.7 & Difficulty estimating BLH, BL influence anomalously low & - \\
\hline 20140812 & 2 & 17.4 & & incl. \\
\hline 20140812 & 3 & 35.4 & & incl. \\
\hline 20140813 & 1 & 29.8 & Large variability in $\mathrm{FT} \mathrm{CH}_{4}$ & - \\
\hline 20140813 & 2 & 42.3 & Large variability in $\mathrm{FT} \mathrm{CH}_{4}$ & - \\
\hline 20140814 & 1 & 7.6 & Difficulty estimating BLH & - \\
\hline 20140814 & 2 & 9.1 & & incl. \\
\hline 20140814 & 3 & 31.6 & & incl. \\
\hline 20140903 & 1 & 1.6 & Large variability in $\mathrm{FT} \mathrm{CH}_{4}$ & - \\
\hline$\ldots$ & $\cdots$ & $\cdots$ & $\cdots$ & $\cdots$ \\
\hline
\end{tabular}


Table S3: (cont.)

\begin{tabular}{|c|c|c|c|c|}
\hline YYYYMMDD & Profile \# & Flux $\left(\mathrm{mg} \mathrm{m}^{-2} \mathrm{~d}^{-1}\right)$ & Observer Information & Final Analysis \\
\hline$\ldots$ & $\ldots$ & $\ldots$ & $\ldots$ & $\ldots$ \\
\hline 20140903 & 2 & 12.8 & & incl. \\
\hline 20140904 & 1 & 52.5 & Large variability in $\mathrm{FT} \mathrm{CH}_{4}$ & - \\
\hline 20140904 & 2 & 30.1 & & incl. \\
\hline 20140904 & 3 & 12.0 & & incl. \\
\hline 20140905 & 1 & 0.7 & Large variability in $\mathrm{FT} \mathrm{CH}_{4}$ & - \\
\hline 20140905 & 2 & -3.0 & Large variability in $\mathrm{FT} \mathrm{CH}_{4}$ & - \\
\hline 20140906 & 1 & 9.8 & Difficulty estimating BLH, BL influence anomalously low & - \\
\hline 20140906 & 2 & 3.7 & & incl. \\
\hline 20140906 & 3 & 22.7 & Large variability in $\mathrm{FT} \mathrm{CH}_{4}$ & - \\
\hline 20140907 & 1 & 23.0 & & incl. \\
\hline 20140907 & 2 & 0.6 & Large variability in $\mathrm{FT} \mathrm{CH}_{4}$ & - \\
\hline 20140907 & 3 & 453.4 & Difficulty estimating BLH, BL influence anomalously low & - \\
\hline 20140909 & 1 & 3.7 & Difficulty estimating BLH, BL influence anomalously low & - \\
\hline 20140911 & 1 & 5.5 & Difficulty estimating BLH & - \\
\hline 20140911 & 2 & 6.2 & Difficulty estimating BLH & - \\
\hline 20140911 & 3 & 3.0 & Difficulty estimating BLH & - \\
\hline$\cdots$ & $\cdots$ & $\cdots$ & $\cdots$ & .. \\
\hline
\end{tabular}


Table S3: (cont.)

\begin{tabular}{|c|c|c|c|c|}
\hline YYYYMMDD & Profile \# & Flux $\left(m g m^{-2} d^{-1}\right)$ & Observer Information & Final Analysis \\
\hline$\ldots$ & $\ldots$ & $\ldots$ & $\ldots$ & $\ldots$ \\
\hline 20140911 & 4 & 16.8 & & incl. \\
\hline 20140911 & 5 & 12.2 & Difficulty estimating BLH & - \\
\hline 20141002 & 1 & 15.4 & & incl. \\
\hline 20141002 & 2 & 11.0 & Large variability in $\mathrm{FT} \mathrm{CH}_{4}$ & - \\
\hline 20141003 & 1 & 4.8 & & incl. \\
\hline 20141003 & 2 & 7.7 & Difficulty estimating BLH & - \\
\hline 20141005 & 1 & 3.3 & Large variability in $\mathrm{FT} \mathrm{CH}_{4}$ & - \\
\hline 20141005 & 2 & 14.3 & & incl. \\
\hline 20141006 & 1 & 6.4 & & incl. \\
\hline 20141006 & 2 & 8.5 & & incl. \\
\hline 20141006 & 3 & 8.2 & Large variability in $\mathrm{FT} \mathrm{CH}_{4}$ & - \\
\hline 20141007 & 1 & 19.7 & & incl. \\
\hline 20141007 & 2 & 26.1 & & incl. \\
\hline 20141007 & 3 & 17.1 & & incl. \\
\hline 20141007 & 4 & 8.0 & & incl. \\
\hline 20141009 & 1 & 6.5 & & incl. \\
\hline$\ldots$ & $\ldots$ & $\ldots$ & $\ldots$ & $\ldots$ \\
\hline
\end{tabular}


Table S3: (cont.)

\begin{tabular}{|c|c|c|c|c|}
\hline YYYYMMDD & Profile \# & Flux $\left(\mathrm{mg} \mathrm{m}^{-2} \mathrm{~d}^{-1}\right)$ & Observer Information & Final Analysis \\
\hline$\ldots$ & $\ldots$ & $\ldots$ & $\ldots$ & $\ldots$ \\
\hline 20141009 & 2 & 22.1 & & incl. \\
\hline 20141104 & 1 & 2.0 & & incl. \\
\hline 20141106 & 1 & 1.6 & & incl. \\
\hline 20141106 & 2 & 3.6 & & incl. \\
\hline 20141106 & 3 & 2.6 & & incl. \\
\hline 20141107 & 1 & 2.4 & & incl. \\
\hline 20141107 & 2 & -4.4 & BL influence anomalously low, Large variability in $\mathrm{FT}^{\mathrm{CH}_{4}}$ & - \\
\hline 20141107 & 3 & 31.6 & Difficulty estimating BLH, BL influence anomalously low & - \\
\hline 20141108 & 1 & 10.0 & Difficulty estimating BLH, BL influence anomalously low & - \\
\hline 20141108 & 2 & 8.4 & & incl. \\
\hline 20141109 & 1 & 12.3 & & incl. \\
\hline 20141109 & 2 & 10.6 & & incl. \\
\hline
\end{tabular}




\section{S3 Methodological uncertainties from bootstrapping / Monte Carlo analysis}

Table S4: Methodological 95\% confidence intervals as described in Sect. 3.7

\begin{tabular}{lccc}
\hline Parameter & $\begin{array}{c}\text { Mean } \\
\left(\mathrm{mg} \mathrm{m}^{-2} \mathrm{~d}^{-1}\right)\end{array}$ & $\begin{array}{c}\text { Minimum } \\
\left(\mathrm{mg} \mathrm{m}^{-2} \mathrm{~d}^{-1}\right)\end{array}$ & $\begin{array}{c}\text { Maximum } \\
\left(\mathrm{mg} \mathrm{m}^{-2} \mathrm{~d}^{-1}\right)\end{array}$ \\
\hline Observations & 0.7 & 0.05 & 3.7 \\
Model initial conditions & 1.2 & 0.05 & 8 \\
$h$ for integration & 0.9 & 0.05 & 4 \\
Free tropospheric $\left[\mathrm{CH}_{4}\right]$ & 3.7 & 0.2 & 14 \\
All of the above & 5.2 & 0.6 & 15
\end{tabular}

\section{S4 Monthly fluxes and uncertainties}

As discussed in the main text, the monthly average $\mathrm{CH}_{4}$ fluxes shown in Fig. 4 are calculated by averaging the individual estimates of $\mathrm{CH}_{4}$ flux into monthly bins. The averaging is adjusted by weighting each individual estimate by the column integrated total surface influence, such that estimates which have a larger degree of spatial coverage are weighted more heavily. In the following tables, the monthly average fluxes are tabulated for reference. In adjacent columns, uncertainties in the monthly average are calculated in four different ways: weighted average uncertainty, the $95 \%$ C.I. for each individual estimate is averaged for each month and weighted by the column integrated total surface influence; average uncertainty, the $95 \%$ C.I. for each individual estimate is averaged for each month with no weighting; weighted standard deviation, the standard deviation of all $\mathrm{CH}_{4}$ flux estimates within a given month is calculated, weighting each residual by the footprint and the square of the $95 \%$ C.I.; and standard deviation, the normal standard deviation is calculated from the residuals of the weighted monthly mean. To be more explicit about the weighted standard deviation, for each month the set of residuals $\left(\hat{\mathrm{E}}_{i}-\overline{\mathrm{E}}\right)$ is weighted by:

$$
w_{i}=\frac{\Delta \mathrm{I}}{\text { C.I }_{i}^{2}}
$$

where $\Delta \mathrm{I}$ is the column integrated surface influence and C.I. $i$ is the $95 \%$ C.I. of the $i^{\text {th }} \mathrm{CH}_{4}$ flux estimate. The weighted standard deviation is then calculated according to:

$$
\sigma=\sqrt{\frac{N \sum_{i} w_{i}\left(\hat{\mathrm{E}}_{i}-\overline{\mathrm{E}}\right)^{2}}{(N-1) \sum_{i} w_{i}}}
$$

where $N$ is the number of profiles for a given month. At the end of each table, the integrated May-September budgets are presented as well as the propagated uncertainty using each method listed above. 
Table S5: CARVE 2012 monthly averaged $\mathrm{CH}_{4}$ fluxes and uncertainties $\left(\mathrm{mg} \mathrm{m}^{-2} \mathrm{~d}^{-1}\right)$ are presented.

\begin{tabular}{|c|c|c|c|c|c|}
\hline Month & $\mathrm{CH}_{4}$ Flux & $\begin{array}{c}\text { Uncertainty } \\
\text { (weighted average) }\end{array}$ & $\begin{array}{l}\text { Uncertainty } \\
\text { (average) }\end{array}$ & $\begin{array}{l}\text { Standard Deviation } \\
\text { (weighted) }\end{array}$ & Standard Deviation \\
\hline 1 & - & - & - & - & - \\
\hline 2 & - & - & - & - & - \\
\hline 3 & - & - & - & - & - \\
\hline 4 & - & - & - & - & - \\
\hline 5 & 9.8 & 3.1 & 4.4 & 2.9 & 3.5 \\
\hline 6 & 12.1 & 3.5 & 4.4 & 7.6 & 11.8 \\
\hline 7 & 22.2 & 8.4 & 11.3 & 13.8 & 11.5 \\
\hline 8 & 14.3 & 4.9 & 6.1 & 2.8 & 4.5 \\
\hline 9 & 11.3 & 3.9 & 4.8 & 4.9 & 9.0 \\
\hline 10 & - & - & - & - & - \\
\hline 11 & - & - & - & - & - \\
\hline 12 & - & - & - & - & - \\
\hline $\begin{array}{c}\text { Budgets \& Uncertainty } \\
\text { (Tg): }\end{array}$ & 2.2 & 0.4 & 0.5 & 0.5 & 0.6 \\
\hline
\end{tabular}


Table S6: CARVE 2013 monthly averaged $\mathrm{CH}_{4}$ fluxes and uncertainties $\left(\mathrm{mg} \mathrm{m} \mathrm{m}^{-2} \mathrm{~d}^{-1}\right)$ are presented.

\begin{tabular}{|c|c|c|c|c|c|}
\hline Month & $\mathrm{CH}_{4}$ Flux & $\begin{array}{c}\text { Uncertainty } \\
\text { (weighted average) }\end{array}$ & $\begin{array}{l}\text { Uncertainty } \\
\text { (average) }\end{array}$ & $\begin{array}{l}\text { Standard Deviation } \\
\text { (weighted) }\end{array}$ & Standard Deviation \\
\hline 1 & - & - & - & - & - \\
\hline 2 & - & - & - & - & - \\
\hline 3 & - & - & - & - & - \\
\hline 4 & 4.0 & 4.5 & 5.2 & 3.1 & 3.9 \\
\hline 5 & 4.2 & 2.2 & 2.0 & 1.6 & 1.9 \\
\hline 6 & 6.5 & 2.1 & 2.7 & 3.5 & 4.0 \\
\hline 7 & 9.3 & 3.9 & 3.6 & 4.5 & 6.3 \\
\hline 8 & 22.4 & 5.0 & 4.5 & 10.1 & 11.6 \\
\hline 9 & 15.6 & 2.6 & 2.4 & 4.7 & 5.6 \\
\hline 10 & 5.0 & 3.3 & 4.7 & 2.7 & 5.4 \\
\hline 11 & - & - & - & - & - \\
\hline 12 & - & - & - & - & - \\
\hline $\begin{array}{c}\text { Budgets \& Uncertainty } \\
\text { (Tg): }\end{array}$ & 1.9 & 0.2 & 0.2 & 0.4 & 0.5 \\
\hline
\end{tabular}


Table S7: CARVE 2014 monthly averaged $\mathrm{CH}_{4}$ fluxes and uncertainties $\left(\mathrm{mg} \mathrm{m} \mathrm{m}^{-2} \mathrm{~d}^{-1}\right)$ are presented.

\begin{tabular}{|c|c|c|c|c|c|}
\hline Month & $\mathrm{CH}_{4}$ Flux & $\begin{array}{c}\text { Uncertainty } \\
\text { (weighted average) }\end{array}$ & $\begin{array}{l}\text { Uncertainty } \\
\text { (average) }\end{array}$ & $\begin{array}{l}\text { Standard Deviation } \\
\text { (weighted) }\end{array}$ & Standard Deviation \\
\hline 1 & - & - & - & - & - \\
\hline 2 & - & - & - & - & - \\
\hline 3 & - & - & - & - & - \\
\hline 4 & - & - & - & - & - \\
\hline 5 & 13.7 & 6.3 & 7.6 & 4.1 & 6.9 \\
\hline 6 & 8.8 & 3.6 & 4.1 & 5.6 & 5.3 \\
\hline 7 & 18.7 & 7.7 & 8.0 & 11.3 & 10.4 \\
\hline 8 & 16.0 & 4.7 & 9.1 & 6.5 & 13.8 \\
\hline 9 & 15.4 & 6.5 & 7.3 & 9.1 & 8.5 \\
\hline 10 & 12.5 & 5.8 & 6.3 & 5.0 & 6.9 \\
\hline 11 & 4.0 & 1.9 & 3.7 & 1.9 & 4.3 \\
\hline 12 & - & - & - & - & - \\
\hline $\begin{array}{c}\text { Budgets \& Uncertainty } \\
\text { (Tg): }\end{array}$ & 2.3 & 0.4 & 0.5 & 0.6 & 0.7 \\
\hline
\end{tabular}




\section{S5 Constraining the $\mathrm{CH}_{4}$ background}

In order to estimate the regional $\mathrm{CH}_{4}$ flux from the aircraft observations presented in the main text, it is necessary to first estimate the natural background of $\mathrm{CH}_{4}$ in the atmosphere. To achieve this, the CARVE flight patterns were planned to include flying up to $5-6 \mathrm{~km}$ a.g.l. so as to sample air well above the planetary boundary layer and any local influence. For each of the resulting profiles of measured $\mathrm{CH}_{4}$ mixing ratios we make the assumption that the average mixing ratio observed in the layer from $500-1500 \mathrm{~m}$ above the top of the boundary layer is representative of the background $\mathrm{CH}_{4}$ mixing ratio in the boundary layer. To assess the accuracy of this claim, we compare these averages to $\mathrm{CH}_{4}$ backgrounds observed in the Alaskan boundary layer from two ground stations. These comparisons are shown in Figure 1 and contrast the background $\mathrm{CH}_{4}$ we estimate from free tropospheric observations, the background $\mathrm{CH}_{4}$ observed at Barrow, Alaska (Dlugogencky, 2016), and the background $\mathrm{CH}_{4}$ derived from particle simulations and observations at the CARVE tower near Fairbanks, Alaska (Karion et al., 2016). These two sites have the advantage that they are situated in the two ecosystems of interest to the current study and have coverage across the entire campaign (with the exception of the Barrow tower in 2012). Overall we can clearly observe that the backgrounds we estimate from free tropospheric observations are certainly within the observations of both ground stations. It is useful to highlight that a latitudinal gradient in $\mathrm{CH}_{4}$ has been observed across the ESRL network, and as our flights cover a wide latitudinal range, it is not surprising that monthly variability (shown as the shaded area around the CARVE aircraft observations) covers the difference between Fairbanks and Barrow.

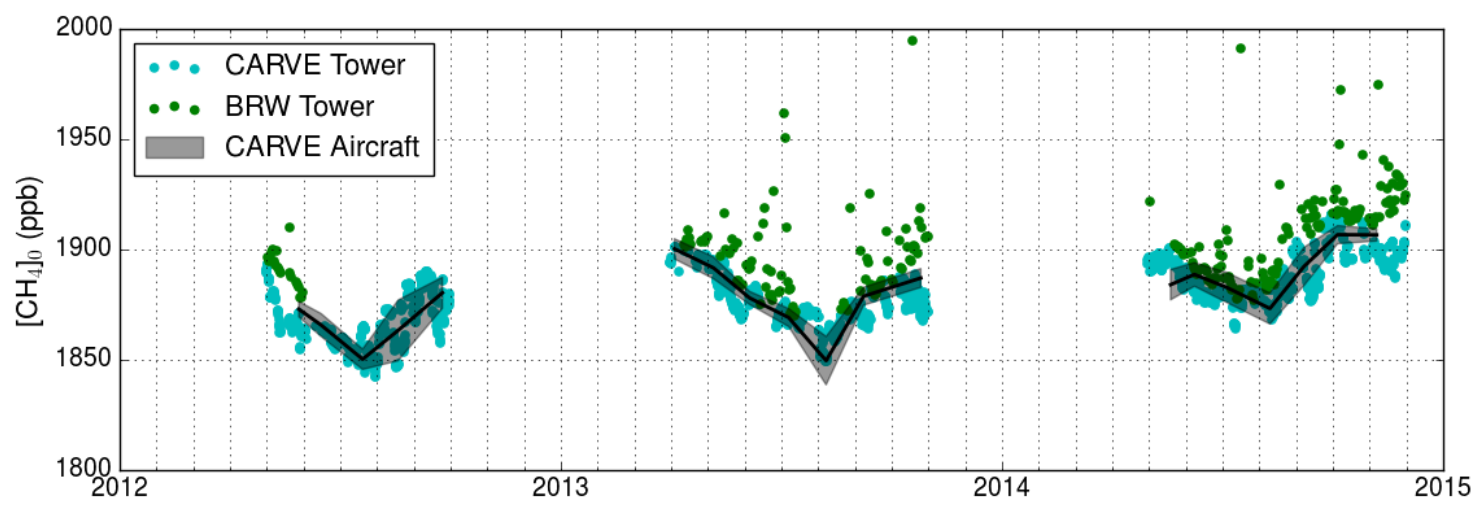

Figure S3: Comparison of monthly average free tropospheric mixing ratios of $\mathrm{CH}_{4}$ observed during the CARVE campaign to boundary layer background $\mathrm{CH}_{4}$ observed at Barrow, Alaska (Dlugogencky, 2016) and derived from observations at the CARVE Tower near Fairbanks Alaska (Karion et al., 2016).

\section{References}

Dlugogencky, E.: Trends in Atmospheric Methane, Tech. rep., National Oceanic and Atmospheric Administration, URL http://www.esrl.noaa.gov/gmd/ccgg/trends_ch4/, date accessed: 01/07/2016, 2016.

Gerbig, C., Lin, J. C., Wofsy, S. C., Daube, B. C., Andrews, A. E., Stephens, B. B., Bakwin, P. S., and Grainger, C. A.: Toward constraining regional-scale fluxes of $\mathrm{CO} 2$ with atmospheric observations over a continent: 2. Analysis of COBRA data using a receptor-oriented framework, J. Geophys. Res., 108, n/a-n/a, doi:10.1029/2003JD003770, URL http://dx.doi.org/10.1029/2003JD003770, 4757, 2003.

Karion, A., Sweeney, C., Miller, J. B., Andrews, A. E., Commane, R., Dinardo, S., Henderson, J. M., Lindaas, J., Lin, J. C., Luus, K. A., Newberger, T., Tans, P., Wofsy, S. C., Wolter, S., and Miller, C. E.: Investigating Alaskan methane and carbon dioxide fluxes using measurements from the CARVE tower, Atmos. Chem. Phys., 16, 5383-5398, doi:10.5194/acp-16-5383-2016, URL http://www . atmos-chem-phys.net/16/5383/2016/, 2016. 